\title{
Evaluation of Copeptin level and Peradeniya Score as Predictors of Severity and Outcome in Acute Organophosphorus Pesticides Poisoned Patients Admitted to the Poison Control Center Ain Shams University Hospitals (A Prospective Study)
}

\author{
Manal Ali Abd AL-Kareem, Aya Shawky Khater and Manar Yehia Ali El-Khashab ${ }^{1}$ \\ ${ }^{1}$ Department of Forensic Medicine and Clinical Toxicology, Faculty of Medicine, Ain Shams University, Cairo, Egypt.
}

\begin{abstract}
Background: Acute organophosphorus poisoning (AOPP) is one of the important causes of morbidity and mortality especially in developing countries. Exposure to organophosphorus (OP) compounds leads to inhibition of cholinesterase enzyme, resulting in overstimulation of muscarinic and nicotinic receptors. Hence, central, peripheral and autonomic manifestations may lead to death. The lack of valid scoring systems for classification of AOPP severity, render difficult outcome prediction and inaccurate potential complication incidence. There are emerging options for new biochemical markers in AOPP that may facilitate the prediction of severity and/ or outcome. Aim of the work: Evaluation and correlation of the role of serum Copeptin level and Peradeniya organophosphorus poisoning (POP) scale in predicting AOPP severity and outcome in 90 AOPP patients admitted to the Poison Control Center - Ain Shams University Hospitals (PCC-ASUHs). Subjects and Methods: A prospective cross sectional, hospital-based study carried out on 90 AOPP patients admitted to PCCASUHs. The diagnosis was verified by history of OP exposure, initial assessment of clinical manifestations and serum Pseudocholinestrase level measurement. Results: In the current study, according to POP scale $48 \%$ of the patients were mild, $42 \%$ were moderate and $10 \%$ were severe cases. Deaths were reported among $11 \%$ of the cases. Serum Copeptin level was positively associated with the severity and outcome, with cut off level $\geq 3.9 \mathrm{ng} / \mathrm{ml}$ for mortality prediction. POP score was directly proportional with hospital stay duration, serum Copeptin level and the outcome. Hospital disposition, mechanical ventilation and coma were positively associated with the degree of severity. Conclusion: Our study concluded that POP scale can be used as a reliable scoring system for AOPP classification of severity and early detector of hospital disposition either ICU or in- patient, hospital stay duration, total atropine dose, incidence of mechanical ventilation and mortality. Serum Copeptin level is positively associated with AOPP severity and had a role in outcome prediction. Recommendation: Early assessment of AOPP severity by POP score can facilitate categorization of the patient's severity state and predict morbidity and mortality. Serum Copeptin level can be used as a tool for evaluation of severity and prognostic marker for the outcome of AOPP.
\end{abstract}

Key words Acute organophosphorus poisoning (AOPP), Peradeniya organophosphorus poisoning (POP) scale, serum Copeptin.

\section{Introduction}

$\mathrm{O}$ rganophosphorus (OP) compounds have been widely used for decades in agriculture for crop protection and pest control. Its widespread use and easy availability have increased the possibility of poisoning with these compounds (Tripathi, 2014).

Organophosphorus (OP) compounds causes irreversible inhibition of acetylcholinesterase (AChE), leading to persistent stimulation of muscarinic and nicotinic receptors, accounting for various manifestations i.e. miosis, bradycardia, bronchoconstriction, hypotension, diarrhea, tachycardia and skeletal muscle fasciculation. As well as, central nervous system (CNS)related acute effects including anxiety, confusion, 
seizures and central cardio-respiratory paralysis (Pereira et al., 2014).

Atropine can reverse the muscarinic symptoms, cross the blood brain barrier (BBB) and counteracts the effects of Ach accumulation in the CNS. However, it is not effective on nicotinic receptor medicated manifestations (Eddleston \& Chowdhury, 2015).

Cholinestrase (ChE) reactivators known as oximes react with the phosphorus atom of OP attached at the enzyme, then oxime phosphate is formed resulting in reactivated ChE. Also, oximes slow the ageing of the phosphorylated enzyme complex and convert OP to a harmless compound (Zhuang et al., 2016).

Prognostic evaluation and estimation are very much critical and important for the outcome prediction. Hence, various descriptive and prognostic evaluation scales (scoring systems) have been developed and used in the emergency department to predict the severity and mortality rate of OP compounds poisoning. A simple system based on clinical features is likely to be most useful in low-income countries where most of poisoning occurs (Mahdavinejad et al., 2014).

Peradeniya OP poisoning (POP) scale is a simple and effective system to determine the severity AOPP. Common clinical manifestations of poisoning are selected as parameters each is assessed on a three-point scale varying from 0 to 2 . The score is obtained at initial presentation before any medical intervention and it represents the muscarinic, nicotinic and central effects of acute cholinergic manifestations. The overall score of 0 to3 considered as mild poisoning, 4 to 7 as moderate poisoning and 8 to 11 as severe poisoning (Dubey et al., 2016).

Various systems and metabolic disorders (e.g., acid-base disturbances) could be assessed and calculated for the early predication of severity and outcome prognosis in AOPP, where toxins may cause lesions in tissues and multiple organs dysfunctions. Copeptin is considered to be a novel marker that indicate individual stress, Copeptin also has good stability in the plasma, thus used in the prognostic evaluation of diseases that may result in different system disorders $(\mathrm{Wu}$ et al., 2016).

Copeptin is co-synthesized with arginine vasopressin (AVP) in the circulation of critically ill subjects. AVP is one of the stress hormones, as it is associated with corticotropic-releasing hormone (CRH) leading to production of adrenocorticotropic hormone (ACTH) and cortisol. Serum cortisol is proportional to and reflects stress levels and can predict prognostic outcome in different diseases (Hu et al., 2015).

Thus, Copeptin mirrors AVP concentration and can be used as a replacement biomarker of AVP as the measurement of AVP is challenging since AVP is an unstable molecule and it is mainly bound to platelets. In advanced and acute heart failure or in terms of lifethreatening conditions, such as severe sepsis, hemorrhagic shock, ischemic stroke or acute myocardial infarction (AMI), Copeptin raises above hundred folds (Dobša \& Edozien, 2013; Jiang et al., 2015).

\section{Aim of the work}

The aim of the current study was to Evaluate and correlate the role of serum Copeptin level and Peradeniya organophosphorus poisoning (POP) scale in predicting AOPP severity and outcome in 90 AOPP patients admitted to the Poison Control Center - Ain Shams University Hospitals (PCC-ASUHs).

\section{Subjects and Methods}

This work presented a prospective cross-sectional hospital-based study assessed the potential role of measuring serum Copeptin level and applying Peradeniya organophosphorus poisoning (POP) scale in predicting AOPP the severity and the outcome in 90 AOPP patients admitted to (PCC-ASUHs) during the period from November 2016 till March 2018. The socio-demographic data, intoxication data, classification and clinical examination according to POP score, laboratory parameters (serum Pseudocholinestrase, serum Na, serum $\mathrm{K}$ and serum Copeptin), hospital disposition, total dose of atropine as well as outcome for all patients were recorded.

All the obtained data was recorded in a special observational sheet then statistically analyzed and presented in tables and diagrams.

\section{Ethical Consideration}

An informed written consent has been obtained from each patient or from his/her caregiver before inclusion in the study for obtaining medical history, clinical examination, taking blood samples for laboratory investigations, keeping the patient's confidentiality by keeping them anonymous. Approval of Ethical Committee and the Director of PCC were also obtained.

\section{Subjects}

90 patients with AOPP were included in the current study and classified according to POP score into mild, moderate and severe.

\begin{tabular}{|l|l|l|}
\hline \multirow{4}{*}{ Pupil size } & $>2 \mathrm{~mm}$ & 0 \\
\cline { 2 - 3 } & $<2 \mathrm{~mm}$ & 1 \\
\cline { 2 - 3 } Respiratory & Pinpoint & 2 \\
\hline \multirow{3}{*}{ rate } & $<20 / \mathrm{min}$ & 0 \\
\cline { 2 - 3 } & $\geq 20 / \mathrm{min}$ & 1 \\
\cline { 2 - 3 } & $\geq 20 / \mathrm{min}$ with central cyanosis & 2 \\
\hline \multirow{4}{*}{ Fasciculation rate } & $>60 / \mathrm{min}$ & 0 \\
\cline { 2 - 3 } & $41-60 / \mathrm{min}$ & 1 \\
\cline { 2 - 3 } & $<40 / \mathrm{min}$ & 2 \\
\cline { 2 - 3 } & None & 0 \\
\cline { 2 - 3 } Level of & generalized or continuous & 1 \\
\hline \multirow{3}{*}{ Conscious } & Conscious and rationale & 2 \\
\cline { 2 - 3 } & Impaired response to verbal commands & 1 \\
\cline { 2 - 3 } Seizures & No response to verbal & 2 \\
\hline & Absent & 0 \\
\cline { 2 - 3 } & Present & 1 \\
\hline
\end{tabular}

Peradeniya Organophosphorus Poisoning (POP) Scale (cited in Dubey et al., 2016). 


\section{Exclusion criteria}

Patients with present history of acute myocardial infarction or heart failure. Patients with history of liver dysfunction.

Patients who received atropine and/or oximes prior to PCC presentation. Patients with history of co-ingestion of toxins other than OP compounds. Patients presented to PCC with delay time more than 24 hours. Patients less than 18 years old.

\section{Methods}

History taking, examination and investigations were done for each patient and recorded in a special sheet designed for the study.

The following data for each patient was obtained:

\section{Clinical data:}

Clinical evaluation of the patients was carried out on arrival to the emergency room (ER) of PCC-ASUHs regarding general and systemic examination.

\section{Laboratory data:}

Blood sample of $5 \mathrm{ml}$ was collected under complete sterile conditions by a single prick on admission, after initial resuscitation and stabilization of patient and, for biochemical analysis:

- $\quad$ Serum electrolytes: Serum sodium $\left(\mathrm{Na}^{+}\right)$and potassium $(\mathrm{K}+)$ level.

- $\quad$ Serum ABGs: $\left(\mathrm{PH}, \mathrm{PCo}_{2}, \mathrm{PO}_{2}, \mathrm{HCO}_{3}\right)$.

- $\quad$ Serum Pseudocholinesterase level.

- $\quad$ Serum Copeptin level.

III. Treatment and hospital admission: Treatment measures received during hospital stay including decontamination methods, atropine, oximes and any other symptomatic treatment needed were given according to the patient condition and according to the protocol of management in PCC-ASUHs.

Outcome: All the patients were observed for short-term outcomes; that they were either discharged or died and the total duration of hospital stay.

Complications: All patients observed for the complications developed during hospital stay e.g. intermediate syndrome (IMS), need for ICU admission, hospital stay duration, need for mechanical ventilation and coma.

Statistical analysis

The obtained data were revised, coded and organized for statistical analysis using SPSS (Statistical package for Social Science) version (18) software (SPSS Inc, USA). Data were presented and suitable analysis was done according to the type of data obtained for each parameter.

\section{Results}

The present study was performed at Poison Control Center of Ain Shams University hospitals (PCC ASUHs) during the period from November 2016 till March 2018. This work presents a prospective crosssectional study that included 90 patients admitted at PCC-ASUHs with AOPP. Their personal history (sociodemographic data), intoxication history, classification and clinical examination according to POP score. As well as laboratory parameters (serum Pseudocholinestrase, serum $\mathrm{Na}$, serum $\mathrm{K}$ and serum Copeptin), hospital disposition, atropine dosage received as well as outcome for all patients were recorded.

Table (1): Chi-Square statistical analysis test comparing gastrointestinal tract (GIT), respiratory and neurological manifestations among mild, moderate and severe patients in the present study.

\begin{tabular}{|c|c|c|c|c|c|c|c|c|c|c|c|c|}
\hline \multirow{3}{*}{\multicolumn{3}{|c|}{ Manifestations }} & \multicolumn{8}{|c|}{ POP Score } & \multirow{2}{*}{\multicolumn{2}{|c|}{ Chi-Square }} \\
\hline & & & \multicolumn{2}{|c|}{ Mild } & \multicolumn{2}{|c|}{ Moderate } & \multicolumn{2}{|c|}{ Severe } & \multicolumn{2}{|c|}{ Total } & & \\
\hline & & & $\mathbf{N}$ & $\%$ & $\mathbf{N}$ & $\%$ & $\mathbf{N}$ & $\%$ & $\mathbf{N}$ & $\%$ & $\mathbf{X}^{2}$ & $\mathbf{P}$ \\
\hline \multirow[t]{3}{*}{ GIT } & \multicolumn{2}{|l|}{ Vomiting } & 43 & 100.0 & 38 & 100.0 & 9 & 100.0 & 90 & 100.0 & & \\
\hline & \multirow{2}{*}{\multicolumn{2}{|c|}{$\begin{array}{l}\text { Diarrhea } \\
\text { Abdominal Colic }\end{array}$}} & 25 & 58.14 & 29 & 76.32 & 9 & 100.0 & 63 & 70.00 & 7.459 & 0.024 \\
\hline & & & 41 & 95.35 & 35 & 92.11 & 6 & 66.67 & 82 & 91.11 & 7.640 & 0.022 \\
\hline \multirow[t]{3}{*}{ Respiratory } & \multicolumn{2}{|c|}{ Respiratory distress } & 3 & 6.98 & 25 & 65.79 & 9 & 100.0 & 37 & 41.11 & 43.146 & $<0.001$ \\
\hline & \multicolumn{2}{|l|}{ Crepitation } & 7 & 16.28 & 30 & 78.95 & 9 & 100.0 & 46 & 51.11 & 41.271 & \\
\hline & \multicolumn{2}{|l|}{ Apnea } & 0 & 0.00 & 0 & 0.00 & 1 & 11.11 & 1 & 1.11 & 9.101 & 0.011 \\
\hline \multirow[t]{7}{*}{ Neurological } & \multicolumn{2}{|l|}{ Seizures } & 0 & 0.00 & 0 & 0.00 & 1 & 11.11 & 1 & 1.11 & 9.101 & 0.011 \\
\hline & \multirow[t]{3}{*}{$\begin{array}{l}\text { Conscious } \\
\text { level }\end{array}$} & $\begin{array}{l}\text { Conscious \& } \\
\text { rational }\end{array}$ & 42 & 97.67 & 12 & 31.58 & 0 & 0.00 & 54 & 60.00 & \multirow[t]{3}{*}{81.749} & \multirow[t]{6}{*}{$<0.001$} \\
\hline & & $\begin{array}{l}\text { Impaired verbal } \\
\text { response }\end{array}$ & 1 & 2.33 & 18 & 47.37 & 0 & 0.00 & 19 & 21.11 & & \\
\hline & & $\begin{array}{l}\text { No verbal } \\
\text { response }\end{array}$ & 0 & 0.00 & 8 & 21.05 & 9 & 100.0 & 17 & 18.89 & & \\
\hline & \multirow[t]{3}{*}{ Fasciculation } & None & 38 & 88.37 & 11 & 28.95 & 0 & 0.00 & 49 & 54.44 & \multirow[t]{3}{*}{74.480} & \\
\hline & & $\begin{array}{l}\text { Continuous or } \\
\text { generalized }\end{array}$ & 5 & 11.63 & 22 & 57.89 & 1 & 11.11 & 28 & 31.11 & & \\
\hline & & $\begin{array}{l}\text { Continuous and } \\
\text { generalized }\end{array}$ & 0 & 0.00 & 5 & 13.16 & 8 & 88.89 & 13 & 14.44 & & \\
\hline
\end{tabular}

X2: Chi-square statistical analysis test ,POP: Peradeniya Organophosphorus Poisoning, GIT: Gastrointestinal tract, $P$ $>0.05$ : non- significant, $P<0.05$ : Significant, $P<0.01$ : Highly Significant 
Table (2): Chi-Square statistical analysis test of the arterial blood gases (ABGs), serum Na and serum K among mild, moderate and severe groups of patients in the present study.

\begin{tabular}{|c|c|c|c|c|c|c|c|c|c|c|c|}
\hline \multirow{3}{*}{\multicolumn{2}{|c|}{ Laboratory parameter }} & \multicolumn{8}{|c|}{ POP Score } & \multirow{2}{*}{\multicolumn{2}{|c|}{ Chi-Square }} \\
\hline & & \multicolumn{2}{|c|}{ Mild } & \multicolumn{2}{|c|}{ Moderate } & \multicolumn{2}{|c|}{ Severe } & \multicolumn{2}{|c|}{ Total } & & \\
\hline & & $\mathbf{N}$ & $\%$ & $\mathbf{N}$ & $\%$ & $\mathbf{N}$ & $\%$ & $\mathbf{N}$ & $\%$ & $\mathrm{X}^{2}$ & P-value \\
\hline \multirow[t]{5}{*}{ ABGs interpretation } & Normal & 34 & 79.07 & 16 & 42.11 & 1 & 11.11 & 51 & 56.67 & \multirow[t]{5}{*}{43.494} & \multirow[t]{5}{*}{$<0.001$} \\
\hline & Respiratory acidosis & 8 & 18.60 & 17 & 44.74 & 3 & 33.33 & 28 & 31.11 & & \\
\hline & Metabolic acidosis & 1 & 2.33 & 3 & 7.89 & 1 & 11.11 & 5 & 5.56 & & \\
\hline & Mixed acidosis & 0 & 0.00 & 1 & 2.63 & 4 & 44.44 & 5 & 5.56 & & \\
\hline & Respiratory alkalosis & 0 & 0.00 & 1 & 2.63 & 0 & 0.00 & 1 & 1.11 & & \\
\hline \multirow{2}{*}{$\begin{array}{l}\text { Serum Na } \\
(\mathrm{mEq} / \mathrm{L})\end{array}$} & Normonatremia & 38 & 88.37 & 29 & 76.32 & 5 & 55.56 & 72 & 80.00 & \multirow[t]{2}{*}{5.567} & \multirow[t]{4}{*}{$>0.05$} \\
\hline & Hyponatremia & 5 & 11.63 & 9 & 23.68 & 4 & 44.44 & 18 & 20.00 & & \\
\hline \multirow[t]{2}{*}{ Serum K (mEq/L) } & Normokalemia & 25 & 58.14 & 23 & 60.53 & 3 & 33.33 & 51 & 56.67 & \multirow[t]{2}{*}{2.264} & \\
\hline & Hypokalemia & 18 & 41.86 & 15 & 39.47 & 6 & 66.67 & 39 & 43.33 & & \\
\hline
\end{tabular}

Na: Sodium, K: Potassium, X2: Chi-square statistical analysis test, POP: Peradeniya Organophosphorus Poisoning, $P$ $>0.05$ : Non-Significant , $P<0.05$ : Significant, $P<0.01$ : Highly Significant

Table (3): One-way ANOVA and TUKEY'S Test statistical analysis of serum Na, serum K levels, serum Pseudocholinestrase (PChE) and serum Copeptin among mild, moderate and severe patients included in the present study.

\begin{tabular}{|c|c|c|c|c|c|c|c|c|c|c|c|c|c|c|c|c|}
\hline \multirow{2}{*}{\multicolumn{2}{|c|}{$\begin{array}{l}\text { Laboratory } \\
\text { parameter }\end{array}$}} & \multirow{3}{*}{$\begin{array}{l}\text { Total } \\
\text { 122- } \\
145 \\
\end{array}$} & \multicolumn{9}{|c|}{ POP Score } & \multicolumn{2}{|c|}{ ANOVA } & \multicolumn{3}{|c|}{ TUKEY'S Test } \\
\hline & & & \multicolumn{3}{|c|}{ Mild } & \multicolumn{3}{|c|}{ Moderate } & \multicolumn{3}{|c|}{ Severe } & \multirow{3}{*}{$\begin{array}{c}\mathbf{F} \\
\\
\\
5.80 \\
0\end{array}$} & \multirow{3}{*}{$\begin{array}{l}\text { P- } \\
\text { valu } \\
\text { e }\end{array}$} & \multirow{3}{*}{$\begin{array}{l}\begin{array}{l}\text { Mild } \\
\& \\
\text { mode } \\
\text { rate }\end{array} \\
\\
0.186\end{array}$} & \multirow{3}{*}{$\begin{array}{l}\begin{array}{l}\text { Mil } \\
\text { d\& } \\
\text { Sev } \\
\text { ere }\end{array} \\
\begin{array}{l}0.00 \\
4\end{array}\end{array}$} & \multirow{3}{*}{$\begin{array}{l}\begin{array}{l}\text { Moder } \\
\text { ate \& } \\
\text { severe }\end{array} \\
0.078\end{array}$} \\
\hline \multirow{2}{*}{$\begin{array}{l}\text { Serum } \\
\text { Na } \\
(\mathrm{mEq} / \\
\mathrm{L})\end{array}$} & $\begin{array}{l}\text { Ran } \\
\text { ge }\end{array}$ & & 131 & - & 144 & 126 & - & 147 & 122 & - & 141 & & & & & \\
\hline & $\begin{array}{l}\text { Me } \\
\text { an } \\
\pm \text { SD }\end{array}$ & $\begin{array}{l}137.3 \\
78 \\
\pm 4.49 \\
\quad 9\end{array}$ & $\begin{array}{l}138 . \\
605\end{array}$ & \pm & 3.431 & $\begin{array}{l}136 . \\
921\end{array}$ & \pm & $\begin{array}{c}4.05 \\
0\end{array}$ & $\begin{array}{l}133 \\
.44 \\
4\end{array}$ & \pm & $\begin{array}{c}7.81 \\
2\end{array}$ & & & & & \\
\hline \multirow{2}{*}{$\begin{array}{l}\text { Serum } \\
\text { K } \\
(\mathrm{mEq} / \\
\mathrm{L})\end{array}$} & $\begin{array}{l}\text { Ran } \\
\text { ge }\end{array}$ & $2.3-5$ & 2.3 & - & 4.5 & 2.6 & - & 5 & 2.4 & - & 4.4 & \multirow[b]{2}{*}{$\begin{array}{c}2.47 \\
1\end{array}$} & \multirow[b]{2}{*}{$\begin{array}{l}0.09 \\
0\end{array}$} & & & \\
\hline & $\begin{array}{l}\text { Me } \\
\text { an } \\
\pm \text { SD } \\
\end{array}$ & $\begin{array}{l}3.517 \\
\pm \\
\mathbf{0 . 4 7 6}\end{array}$ & $\begin{array}{l}3.54 \\
2\end{array}$ & \pm & 0.412 & $\begin{array}{l}3.56 \\
6\end{array}$ & \pm & $\begin{array}{c}0.48 \\
1\end{array}$ & $\begin{array}{l}3.1 \\
89\end{array}$ & \pm & $\begin{array}{c}0.65 \\
5\end{array}$ & & & & & \\
\hline \multirow{2}{*}{$\begin{array}{l}\text { Serum } \\
\text { PChE } \\
\text { (U/L) } \\
4900- \\
11900\end{array}$} & $\begin{array}{l}\text { Ran } \\
\text { ge }\end{array}$ & $\begin{array}{l}250- \\
3587\end{array}$ & 260 & - & 3000 & 360 & - & $\begin{array}{c}358 \\
7\end{array}$ & 250 & - & 1039 & \multirow{2}{*}{$\begin{array}{c}2.91 \\
9\end{array}$} & \multirow{2}{*}{$\begin{array}{l}0.05 \\
9\end{array}$} & & & \\
\hline & $\begin{array}{l}\text { Me } \\
\text { an } \\
\pm \text { SD }\end{array}$ & $\begin{array}{l}1042 . \\
489 \\
\pm 664 . \\
661 \\
\end{array}$ & $\begin{array}{l}1008 \\
.48\end{array}$ & \pm & $\begin{array}{l}665.2 \\
69\end{array}$ & $\begin{array}{l}1183 \\
.053\end{array}$ & \pm & $\begin{array}{l}691 . \\
818\end{array}$ & $\begin{array}{l}611 \\
.44 \\
4\end{array}$ & \pm & $\begin{array}{l}261 . \\
742\end{array}$ & & & & & \\
\hline \multirow{2}{*}{$\begin{array}{l}\text { Serum } \\
\text { Copep } \\
\text { tin } \\
\text { (ng/ml } \\
\text { ) }\end{array}$} & $\begin{array}{l}\text { Ran } \\
\text { ge }\end{array}$ & $\begin{array}{l}1.2- \\
8.5\end{array}$ & 1.2 & - & 2.7 & 2 & - & 4.3 & 4.8 & - & 8.5 & \multirow[b]{2}{*}{$\begin{array}{c}193 . \\
83\end{array}$} & \multirow[b]{2}{*}{$\begin{array}{l}<0.0 \\
01\end{array}$} & \multirow[b]{2}{*}{$\begin{array}{l}<0.00 \\
1\end{array}$} & \multirow[b]{2}{*}{$\begin{array}{l}<0.0 \\
01\end{array}$} & \multirow[b]{2}{*}{$<0.001$} \\
\hline & $\begin{array}{l}\text { Me } \\
\text { an } \\
\pm \text { SD }\end{array}$ & $\begin{array}{l}2.932 \\
\pm 1.52 \\
0\end{array}$ & $\begin{array}{l}1.87 \\
4\end{array}$ & \pm & 0.436 & $\begin{array}{l}3.28 \\
2\end{array}$ & \pm & $\begin{array}{c}0.60 \\
9\end{array}$ & $\begin{array}{l}6.5 \\
11\end{array}$ & \pm & $\begin{array}{c}1.41 \\
4\end{array}$ & & & & & \\
\hline
\end{tabular}

F one- way analysis of variance (ANOVA) test $\quad$ Na: Sodium K: Potassium, SD: standard deviation, POP: Peradeniya Organophosphorus Poisoning, $P>0.05$ : non-significant, $P<0.05$ : significant, $P<0.01$ : highly significant 
Table (4): Independent t- test statistical analysis of serum Na, serum K levels, serum Pseudocholinestrase (PChE) and serum Copeptin among discharged patients and deaths in the present study.

\begin{tabular}{|c|c|c|c|c|c|c|c|c|c|}
\hline \multirow{2}{*}{\multicolumn{2}{|c|}{ Laboratory parameter }} & \multicolumn{6}{|c|}{ Outcome } & \multicolumn{2}{|c|}{ T-Test } \\
\hline & & \multicolumn{3}{|c|}{ Discharged } & \multicolumn{3}{|c|}{ Deaths } & $\mathbf{T}$ & P-value \\
\hline \multirow{2}{*}{ Serum $\mathrm{Na}(\mathrm{mEq} / \mathrm{L})$} & Range & 124 & - & 147 & 122 & - & 140 & \multirow{2}{*}{2.765} & \multirow{2}{*}{0.007} \\
\hline & Mean \pm SD & 137.825 & \pm & 4.068 & 133.800 & \pm & 6.233 & & \\
\hline \multirow{2}{*}{ Serum K(mEq/L) } & Range & 2.3 & - & 5 & 2.4 & - & 4.4 & \multirow{2}{*}{2.129} & \multirow{2}{*}{0.036} \\
\hline & Mean \pm SD & 3.554 & \pm & 0.448 & 3.220 & \pm & 0.609 & & \\
\hline \multirow{2}{*}{$\begin{array}{l}\text { Serum PChE (U/L) } \\
4900-11900\end{array}$} & Range & 260 & - & 3587 & 250 & - & 1039 & \multirow{2}{*}{2.062} & \multirow{2}{*}{0.042} \\
\hline & Mean \pm SD & 1092.663 & \pm & 683.725 & 641.100 & \pm & 252.646 & & \\
\hline \multirow{2}{*}{$\begin{array}{l}\text { Serum Copeptin } \\
\text { (ng/ml) }\end{array}$} & Range & 1.2 & - & 8.5 & 3.2 & - & 8.5 & \multirow{2}{*}{-7.488} & \multirow{2}{*}{$<0.001$} \\
\hline & Mean \pm SD & 2.599 & \pm & 1.127 & 5.600 & \pm & 1.677 & & \\
\hline
\end{tabular}

Na: Sodium, K: Potassium, $t=$ independent $t$ test, $S D$ : standard deviation, $P>0.05$ : non-significant, $P$ <0.05: significant $P<0.01$ : highly significant

Table (5): Sensitivity and specificity of serum Copeptin as early predictor of mortality of patients included in the present study.

\begin{tabular}{|l|l|l|l|l|l|l|}
\hline Cut off & AUC & Sensitivity & Specificity & PPV & NPV & Accuracy \\
\hline$\geq 3.9$ & 0.953 & 90.0 & 90.0 & 98.6 & 52.9 & $95.3 \%$ \\
\hline
\end{tabular}

PPV: Positive Predictive Value, NPV: Negative Predictive Value, AUC: Area under Curve

Table (6): Pearson correlation (r) between serum Copeptin, hospital stay duration (days) and POP score of the patients in the present study.

\begin{tabular}{|l|c|c|}
\hline & \multicolumn{2}{|c|}{ Correlations } \\
\hline & R & POP score \\
\hline Serum Copeptin & 0.803 & P-value \\
\hline Hospital stay duration (Days) & 0.531 & $<0.001$ \\
\hline
\end{tabular}

$r$ : Correlation coefficient, POP: Peradeniya Organophosphorus Poisoning, $p>0.05=$ non-significant

Table (7): One-way ANOVA and TUKEY'S Test statistical analysis of hospital stay duration (days) and total atropine (ampoules) received among mild, moderate and severe the patients in the present study

\begin{tabular}{|c|c|c|c|c|c|c|c|c|c|c|c|c|c|c|c|}
\hline & \multicolumn{9}{|c|}{ POP Score } & \multicolumn{2}{|c|}{ ANOVA } & \multicolumn{3}{|c|}{ TUKEY'S Test } \\
\hline & & \multicolumn{3}{|c|}{ Mild } & \multicolumn{3}{|c|}{ Moderate } & \multicolumn{3}{|c|}{ Severe } & $\mathbf{F}$ & $\begin{array}{c}\mathbf{P}- \\
\text { value }\end{array}$ & $\begin{array}{l}\text { Mild \& } \\
\text { Modera }\end{array}$ & $\begin{array}{c}\text { Mild } \\
\text { \& } \\
\text { Seve }\end{array}$ & $\begin{array}{c}\text { Modera } \\
\text { te \& }\end{array}$ \\
\hline \multirow{2}{*}{$\begin{array}{l}\text { Hospital } \\
\text { stay } \\
\text { duration } \\
\text { (Days) }\end{array}$} & $\begin{array}{l}\text { Ran } \\
\text { ge }\end{array}$ & 1 & - & 7 & 1 & - & 17 & 2 & - & 21 & \multirow{2}{*}{$\begin{array}{c}14.2 \\
5\end{array}$} & \multirow{2}{*}{$\begin{array}{c}<0.00 \\
1\end{array}$} & \multirow{2}{*}{0.010} & \multirow{2}{*}{$\begin{array}{c}<0.00 \\
1\end{array}$} & \multirow{2}{*}{0.005} \\
\hline & $\begin{array}{l}\text { Mea } \\
\text { n } \\
\pm \text { SD }\end{array}$ & $\begin{array}{c}2.23 \\
3\end{array}$ & \pm & $\begin{array}{c}1.0 \\
8\end{array}$ & 4.42 & \pm & $\begin{array}{c}3.53 \\
8\end{array}$ & $\begin{array}{c}8.33 \\
3\end{array}$ & \pm & 7.228 & & & & & \\
\hline \multirow{2}{*}{$\begin{array}{l}\text { Total } \\
\text { atropine } \\
\text { (Ampoul } \\
\text { e) }\end{array}$} & $\begin{array}{l}\text { Ran } \\
\text { ge }\end{array}$ & 2 & - & $\begin{array}{c}20 \\
6\end{array}$ & 7 & - & $\begin{array}{c}104 \\
0\end{array}$ & 56 & - & 2000 & \multirow[b]{2}{*}{$\begin{array}{c}10.0 \\
5\end{array}$} & \multirow{2}{*}{$\begin{array}{c}<0.00 \\
1\end{array}$} & \multirow[b]{2}{*}{0.064} & \multirow{2}{*}{$\begin{array}{c}<0.00 \\
1\end{array}$} & \multirow[b]{2}{*}{0.012} \\
\hline & $\begin{array}{l}\text { Mea } \\
\text { n } \\
\pm \text { SD }\end{array}$ & 26 & \pm & $\begin{array}{c}35 . \\
1\end{array}$ & $\begin{array}{c}142 . \\
2\end{array}$ & \pm & $\begin{array}{c}202 . \\
4\end{array}$ & $\begin{array}{c}392 . \\
5\end{array}$ & \pm & $\begin{array}{c}614.8 \\
74\end{array}$ & & & & & \\
\hline
\end{tabular}


Table (8): Distribution of the patients according severity by POP score and outcome included in the present study.

\begin{tabular}{|l|l|c|c|}
\hline \multicolumn{2}{|l|}{ Patients groups } & Number & Percentage \% \\
\hline \multirow{3}{*}{ POP on admission } & Mild & 43 & 47.78 \\
\cline { 2 - 4 } & Moderate & 38 & 42.22 \\
\cline { 2 - 4 } & Severe & 9 & 10.00 \\
\hline \multirow{2}{*}{ Outcome } & Discharged & 80 & 88.89 \\
\cline { 2 - 4 } & Deaths & 10 & 11.11 \\
\hline
\end{tabular}

POP: Peradeniya Organophosphorus Poisoning

Table (9): Chi-Square statistical analysis test comparing hospital disposition, complication and the outcome among mild, moderate and severe patients in the present study.

\begin{tabular}{|c|c|c|c|c|c|c|c|c|c|c|c|}
\hline & \multicolumn{8}{|c|}{ POP Score } & \multirow{2}{*}{\multicolumn{2}{|c|}{ Chi-Square }} \\
\hline & & \multicolumn{2}{|c|}{ Mild } & \multicolumn{2}{|c|}{ Moderate } & \multicolumn{2}{|c|}{ Severe } & \multicolumn{2}{|c|}{ Total } & & \\
\hline & & $\mathbf{N}$ & $\%$ & $\mathbf{N}$ & $\%$ & $\mathbf{N}$ & $\%$ & $\mathbf{N}$ & $\%$ & $X^{2}$ & P-value \\
\hline \multirow{2}{*}{$\begin{array}{l}\text { Hospital } \\
\text { disposition }\end{array}$} & In-patient & 34 & 79.07 & 4 & 10.53 & 0 & 0.00 & 38 & 42.22 & \multirow{2}{*}{46.158} & \multirow{5}{*}{$<0.001$} \\
\hline & ICU & 9 & 20.93 & 34 & 89.47 & 9 & 100.00 & 52 & 57.78 & & \\
\hline \multirow{4}{*}{ Complication } & None & 41 & 95.35 & 9 & 23.68 & 0 & 0.00 & 50 & 55.56 & 54.460 & \\
\hline & MV & 0 & 0.00 & 13 & 34.21 & 8 & 88.89 & 21 & 23.33 & 37.221 & \\
\hline & Coma & 1 & 2.33 & 26 & 68.42 & 9 & 100.00 & 36 & 40.00 & 51.720 & \\
\hline & IMS & 1 & 2.33 & 4 & 10.53 & 0 & 0.00 & 5 & 5.56 & 3.174 & 0.205 \\
\hline \multirow{2}{*}{ Outcome } & Discharged & 43 & 100.00 & 35 & 92.11 & 2 & 22.22 & 80 & 88.89 & \multirow{2}{*}{46.273} & \multirow{2}{*}{$<0.001$} \\
\hline & Deaths & 0 & 0.00 & 3 & 7.89 & 7 & 77.78 & 10 & 11.11 & & \\
\hline
\end{tabular}

ICU: Intensive care unit, MV: Mechanical ventilation, IMS: Intermediate syndrome, $X^{2}$ Chi-square statistical analysis test POP: Peradeniya Organophosphorus Poisoning

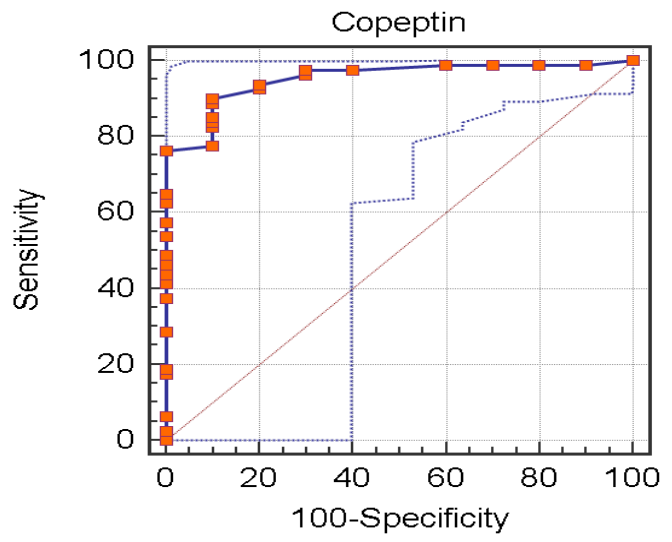

Fig (1): The receiver operating characteristic (ROC) curve of serum Copeptin as early predictor of the outcome

\section{Discussion}

OP compounds are one of the most common causes of poisoning, especially in developing countries. Despite the great developments in the ICU management, OP compounds are still a greatly contributing agents of intoxication with mortality rates reaching up to $10-20 \%$. So early diagnosis and appropriate treatment is often lifesaving (Chowdhary et al., 2014).
Owing to limited availability of resources, not all AOPP patients are managed in ICUs, hence coming the urge for early determination of clinical features and criteria that predict the need for ventilator support to be identified at initial examination. POP scale assesses the severity of the poisoning based on the symptoms at presentation and is a simple way to use and effective to 
determine the need for ventilatory support early on in the course (Tripathi, 2014).

In AOPP, depressed serum PChE level supports the diagnosis of poisoning, however it may not show a significant relationship with severity of poisoning, so there is a need for new biochemical markers to detect severity and prognosis of cases with OP compounds intoxication (Bhattacharyya et al., 2011).

The present study was performed at PCCASUHs. This work represented a cross sectional study including 90 AOPP patients, who were selected according to predetermined criteria, during period from November 2016 till March 2018. This work aimed to evaluate and correlate the role of serum Copeptin level and Peradeniya organophosphorus poisoning (POP) scale in predicting AOPP severity and outcome.

All patients in the current study were classified on admission according to POP scale. Forty-three cases were mild followed by 38 moderate cases and only 9 were severe cases. As regard the outcome of the study, 80 patients were discharged while 10 deaths were recorded. These results were close to (Patel et al., 2016) study that revealed 47 were mild cases, 41 moderate cases and 12 severe cases, meanwhile 91 patients were discharged and 9 patients died.

In the present study, the mean serum $\mathrm{Na}$ level was $137 \pm 4.4(\mathrm{mEq} / \mathrm{L})$, with no significant correlation to severity, these findings were similar to Binny (2017), however, decreased serum $\mathrm{Na}$ level was significantly correlated to the outcome in this study. Regarding serum $\mathrm{K}$ level, hypokalemia was recorded in $43 \%$ of patients, with mean value $3.5 \pm 0.4(\mathrm{mEq} / \mathrm{L})$, with no significant correlation to severity, these results were similar to Banday et al., (2015) results that failed to correlate hypokalemia to severity of OP poisoning. However, mean serum $\mathrm{K}$ level was lower in deaths than in discharged patients, showing significant association between hypokalemia and the outcome, this finding was similar to a study performed by Tripathy et al., (2018) that associated hypokalemia with mortality.

According to Prasad (2014), poor outcome in OP poisoning was noted in patients suffered from respiratory distress with hypokalemia. Also, it was found that there was a remarkable decrease in serum potassium relating to OP toxicity associated with muscle weakness and paralysis finally leading to death.

Hypokalemia and paralysis are potentially reversible medical emergencies, however, when it was coupled with PChE reduction, high morbidity and mortality was observed. In addition, hypokalemia may aggravate muscular weakness due to inhibition ACh by OP poisoning (Tripathy et al., 2018).

The mean serum PChE level in our study was $1042 \pm 664(\mathrm{U} / \mathrm{L})$, with no significant correlation to the grade of severity, however, there was a significant negative correlation between serum PChE level and the outcome, where mean serum PChE level was depressed in deaths more than that among the discharged patients.
In a study conducted by Tripathi (2014), they concluded that low serum PChE level was associated with both higher mortality and higher degree of severity. Also, Muley et al., (2014) agreed with the current study as regard significance correlation between low serum PChE level and bad prognosis.

The mean serum Copeptin level in the present study was $2.9 \pm 1.5(\mathrm{ng} / \mathrm{ml})$, showing significant positive correlation between the degree of severity and mean Copeptin level in each group, also there was a significant positive association between serum Copeptin level and the outcome. The predictive cut - off value of the serum Copeptin was $\geq 3.9(\mathrm{ng} / \mathrm{ml})$, with sensitivity and specificity $90 \%$, these results were close to WU et al., (2016) study that reported the optimum cut -off value of the serum Copeptin was $6.3(\mathrm{ng} / \mathrm{ml})$, with sensitivity and specificity over $90 \%$, hence the capability of serum Copeptin of prediction of the outcome in AOPP patients.

In a study conducted by $\mathrm{Li}$ et al., (2014) demonstrated that AOPP patients have elevated Copeptin levels, as compared with normal subjects, and that its levels increase with increasing poisoning severity.

The hospital stay duration in the current study ranged from one to 21 days with mean 3.7 days, with positive significant correlation between POP score and hospital stay duration. Thus, by applying Pearson correlation, the hospital stay duration was longer in severe group more than in moderate and mild groups. These results were in accordance with Girish et al., (2016) results. This could be explained by that most of severe patients might be vulnerable to variant complications that require prolonged ICU stay (Vernekar \& Shivaraj, 2017).

Hospital disposition in the current study was reported as $42 \%$ were admitted into the in-patient department and 57\% were admitted into the ICU, where there was a positive significant correlation between hospital disposition and the POP score, thus all severe cases and $89 \%$ of moderate cases were admitted into ICU. These results were in accordance with Girish et al., (2016) and Kumar \& Sahna, (2017).

Concerning the complications, $23 \%$ of patients in the current study developed disturbed conscious level, $40 \%$ required mechanical ventilation, while $5 \%$ suffered from IMS. The study showed positive significant correlation between the disturbance of conscious level, the requirement of mechanical ventilation with the degree of severity, these results were in accordance with the results recorded by Kumar et al., (2017).

As regard the outcome of patients, in the current study deaths represented $11.1 \%$ of patients while the remaining $88.8 \%$ were discharged. This agreed with the results reported by Sun et al., (2015). Significant positive correlation was noticed between severity of poisoning according to POP score and death as increased severity was associated with increased mortality, which was in accordance with the results reported by Girish et al., (2016) and Vernekar \& Shivaraj, (2017). 


\section{Conclusion}

Our study concluded that the severity of AOPP can be classified according to POP score, that can predict the site of admission either ICU or inpatient, incidence of mechanical ventilation need, hospital stay duration and outcome of the patients.

Serum PChE, serum $\mathrm{K}$ as well as serum $\mathrm{Na}$ levels have low role in detection of AOPP severity, however they have helpful role in predicting the outcome of the patients.

Serum Copeptin level has a significant role in evaluation of AOPP severity, total atropine demand as well as in prediction of the outcome of patients when compared to serum Na and PChE, with sensitivity and specificity of $90 \%$ and $\geq 3.9(\mathrm{ng} / \mathrm{ml})$ as a cut off value for prediction of mortality.

\section{Recommendations}

- Serum Copeptin level can be used as a tool of evaluation for severity and prognostic marker for the outcome of AOPP.

- POP score can be used to facilitate categorization of severity state of the patients and as a reliable predictor for morbidity and mortality in AOPP.

\section{Limitation of the present study}

1. Identification of OP compounds type could not be available, while OP poisoning was diagnosed based on the history of OP exposure, clinical toxidrome and the measurement of serum Pseudocholinesterase enzyme.

2. Measurement of erythrocyte (true) cholinesterase could not be done because of the non-availability of the test during the study period.

3. Study group was just adequate.

4. Serial estimation of the biochemical parameters during hospital stay was not done.

\section{References}

Banday T, Tathineni B, Desai M, et al., (2015): Predictors of morbidity and mortality in organophosphorus poisoning: A case study in rural hospital in Karnataka, India, North Amer J med Sci; 7(6): 259-65.

Bhattacharyya K, Phaujdar S, Sarker R, et al., (2011): Serum creatine phosphokinase a probable marker of severity in organophosphorus poisoning. Inter J Toxicol;18 (2):117-23.

Binny L (2017): Biochemical abnormalities in OPC poisoning and its prognostic significance. IOSR J Dental Med Sci;16(7):116-9.

Chowdhary SH, Bhattacharyya R \& Banerjee D (2014): Acute organophosphorus poisoning, Clin Chimica Acta; 431: 66-76.

Dobša L \& Edozien K (2013): Copeptin and its potential role in diagnosis and prognosis of various diseases. Biochem Med; 23(2):172-90.
Dubey T, Sudhanshu Y \& Kawre K (2016): Correlation of severity of organophosphorus poisoning as assessed by Peradeniya organophosphorus poisoning scale with serum amylase and CPK level. Inter J Contemporary Med Res;3(9):25347.

Eddleston M \& Chowdhury F (2015): Pharmacological treatment of organophosphorus insecticide poisoning: the old and the (possible) new, Brit $\mathrm{J}$ Clin Pharmacol; 81(3): 462-70.

Girish S, Kalyan \& Reddy Y (2016): To Assess the Severity of Organophosphorus Compound Poisoning Clinically by Using Peradeniya Score. Ind J App Res; 6 (4):617-9.

Hu W, Ni Y, Ma L, et al., (2015): Serum copeptin as a new biomarker in the early diagnosis of decline in renal function of type 2 diabetes mellitus patients. Inter J Clin Exp Med; 8(6):9730-6.

Jiang L, Feng B, Gao D, et al., (2015): Plasma concentrations of copeptin, C-reactive protein and procalcitonin are positively correlated with APACHE II scores in patients with sepsis. J Inter Med Res; 43(2):188-95.

Kumar G, Rao R \& Nrushen P (2017): A study on serum cholinesterase levels as a prognostic marker in organophosphorus poisoning. Asian Pac J Health Sci.; 4(1):91-9.

Kumar R \& Sahna E (2017): Correlation of serum Pseudocholinesterase level and Peradeniya organophosphorus poisoning scale with the severity and in hospital outcome of acute organophosphorus poisoning. Inter $\mathrm{J}$ Contemporary Med Res; 4(8):1702-5.

Li J, Wang R \& He J (2014): Copeptin and ischemia modified albumin expression and significance of early in acute paraquat poisoning. Shi Yong Lin Chuang Yi Xue Za Zhi; 18: 40-3.

Mahdavinejad A, Pajoumand A, Barari B, et al., (2014): Lack of prognostic role for Glasgow coma scale, serum acetylcholinesterase and leukocyte levels in acute organophosphorus toxicological ICU poisoned patients. Life Sci J; 11(8): 563-7.

Muley A, Shah Ch, Lakhani J, et al., (2014): To identify morbidity and mortality predictors in acute organophosphate poisoning. Ind J Crit Care Med; 18(5): 297-300.

Patel P, Patel V, Patel H, et al., (2016): Study of prognostic value of serum and RBC acetyl cholinesterase level in organophosphorus poisoning and its correlation with the outcome. Inter Arch Integrated Med; 3(3): 147-57.

Pereira E, Aracava Y, DeTolla L, et al., (2014): Animal Models That Best Reproduce the Clinical Manifestations of Human Intoxication with Organophosphorus Compounds. J Pharmacol Exp Therap; 350(2): 313-21.

Prasad D (2014): Reduced levels of serum potassium and plasma cholinesterase in acute organophosphate 
poisoning: possible predictive markers. Asia Pac J Med Toxicol; 3: 68-72.

Sun I, Yoon H, \& Lee K (2015): Prognostic factors in cholinesterase inhibitor poisoning. Med Sci Monit; 21:2900-4.

Tripathi Sh (2014): "Prognostic value of Glasgow Coma Scale, Poisoning Severity Score and serum acetylcholinesterase levels in organophosphorus poisoning”. J Evolution Med Dental Sci; 3(13): 3415-22.

Tripathy S, Rout P, Debta N, et al., (2018): Study of clinical profile of organophosphorus poisoning with special reference to electrocardiographic changes and electrolyte derangement. Int J Adv Med;5(1):50-6.
Vernekar P \& Shivaraj K (2017): Peradeniya organophosphorus poisoning scale (POP) as a predictor of respiratory failure and mortality in organophosphorus poisoning. Sch J App Med Sci; 5(5B):1841-4.

Wu X, Xie W, Cheng Y, et al., (2016): Severity and prognosis of acute organophosphorus pesticide poisoning are indicated by $\mathrm{C}$ reactive protein and copeptin levels and APACHE 2 score. J Exp Therap Med; 11(3): 806-10.

Zhuang Q, Young A, Callam C, et al., (2016): Efforts toward treatments against aging of organophosphorus-inhibited acetylcholinesterase. Annul New York Academy Sci; 1374: 94-104.

\section{الملخص العربى}

تقييم مستوى الكوبيتين و بجموع نقاط البيرادينيا كمتنبأت بشدة ونتائج التسمم الحاد بالمبيدات الحشرية العضوية الفوسفورية للمرضى الذين تم ادخالهم بمركز علاج التسمم بمستشفيات جامعة عين شمس

\section{منال علي عبد الكريم و آية شوقي خاطر و منار يحيي علي'}

يعد التسمم الحاد بالمبيدات العضوية الفوسفورية من أحد أهم الأسباب المرضية والمسببة للوفاة خاصة في البلدان النامية حيث يؤدي التعرض للمركبات

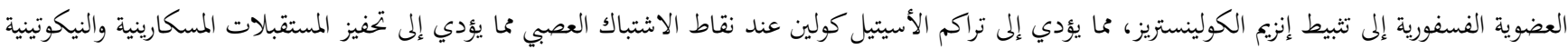
، مما يؤدي إلى مظاهر مركزية وطرفية ولا ارادية قد تؤدي إلى الوفاة.

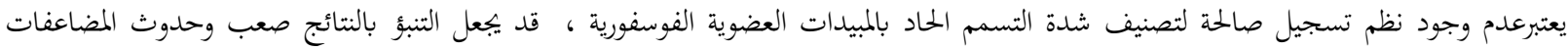

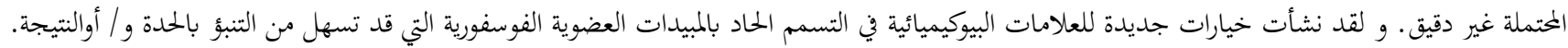

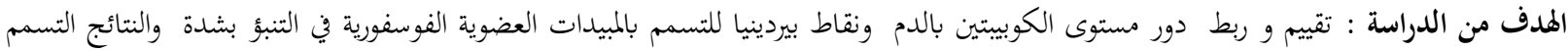

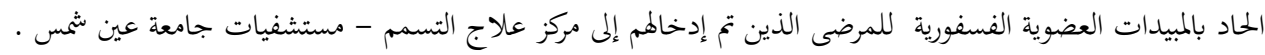

المرضي والطريقة : دراسة مستقبلية مستعرضة ومستندة علي اساس سريري في المستشفى قد تمت على ·و مريض تسمم حاد بالمبيدات العضوية الفسفورية الذين تم حجزهم في مركز علاج التسمم -مستشفيات جامعة عين شمس. وتم التحقق من التشخيص من خلال تاريخ التعرض للمبيد العضوي الفسفوري،

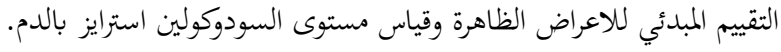

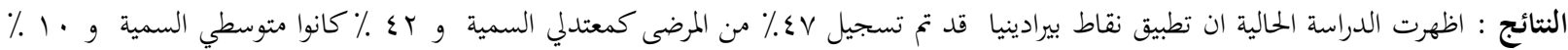

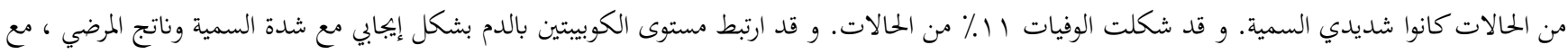

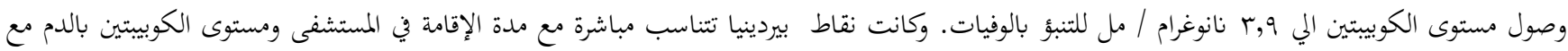

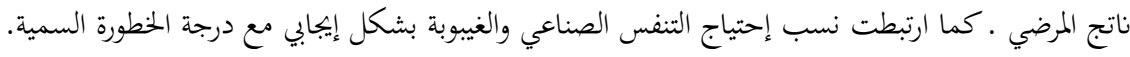

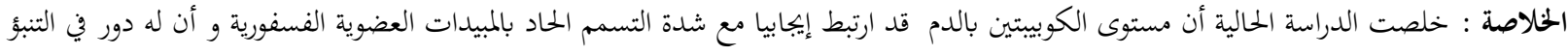

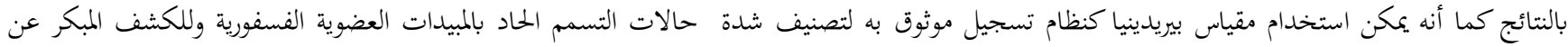

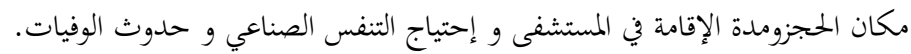
و توصي هذه الدراسة باستخدام مستوي الكوبيتين بالدم كا آداه لتقيم شدة خطورة التسمم الحماد بالمبيدات العضوية الفسفورية ، كما توصي الدراسة باستخدام مقياس بيرادينيا كنظام تسجيل لتصنيف شدة السمية لدي مرضي التسمم الحاد بالمبيدات العضوية الفسفورية.

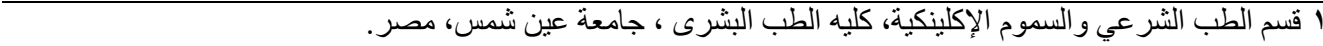

